\title{
A Review of the Evolution of Classical Nova V2676 Oph: Formation of Molecules and Dust Grains
}

\section{Hideyo KAWAKITA*}

Koyama Astronomical Observatory, Kyoto Sangyo University, Motoyama, Kamigamo, Kita-ku, Kyoto 606-8555, Japan

E-mail: kawakthd@cc.kyoto-su.ac.jp

\section{Akira ARAI}

Koyama Astronomical Observatory, Kyoto Sangyo University, Motoyama, Kamigamo, Kita-ku, Kyoto 606-8555, Japan

E-mail: arai6a@cc.kyoto-su.ac.jp

\begin{abstract}
Nova V2676 Oph is the first classical nova in which $\mathrm{C}_{2}$ has been detected during its early phase near the visual-brightness maximum in addition to $\mathrm{CN}$. The presence of $\mathrm{C}_{2}$ and $\mathrm{CN}$ in the nova indicates that its atmosphere was enriched in carbon with $\mathrm{C} / \mathrm{O}>1$. Furthermore, molecule formation in the early phase is likely to be associated with the dust formation that started $\sim 90$ days after the discovery. We have obtained isotopic ratios of carbon and nitrogen for this nova, and they are consistent with model predictions. Based on the lightcurves and optical spectra of the nova, the inferred mass of the white-dwarf component of V2676 Oph is relatively small $\left(\sim 0.6 \mathrm{M}_{\odot}\right)$. The absence of strong [Ne II] emission at $12.8 \mu \mathrm{m}$ and the relatively small ejected mass from V2676 Oph support this hypothesis. However, the mass of the white-dwarf component should be higher $\left(>\sim 1.0 \mathrm{M}_{\odot}\right)$ according to the observed isotopic ratios and theoretical predictions based on thermonuclear runaways.
\end{abstract}

The Golden Age of Cataclysmic Variables and Related Objects IV 11-16 September, 2017

Palermo, Italy

\footnotetext{
* Speaker.
} 


\section{Introduction}

Classical novae are hot objects. They exhibit high effective temperatures $(\sim 8000 \mathrm{~K})$ even at their visual-brightness maxima, when the size of the nova photosphere is at a maximum (and the photospheric temperature is therefore a minimum). Nevertheless, some simple diatomic molecules have been detected in several classical novae with excitation temperatures in the range from $\sim 2500$ $\mathrm{K}$ to $\sim 5000 \mathrm{~K}$ (e.g., $[8,45,36,17]$ and references therein). Spectroscopic observations of molecular bands in novae provide information about isotopic abundances, and observations of the molecules (as intermediate products in the evolution from atomic species to dust grains) are essential for understanding the dust formation in nova outflows.

The classical nova V2676 Oph is unique in that $\mathrm{C}_{2}, \mathrm{CN}$, and $\mathrm{CO}$ molecules - and larger molecules such as polycyclic aromatic hydrocarbons (PAHs) or hydrogenated amorphous carbon (HAC) - have all been detected in the same nova. In addition, significant dust formation began $\sim 90$ days after the nova explosion $[36,51,34,46]$. Dust formation is recognized in about $\sim 20 \%$ of classical novae [59] and usually occurs in "CO novae" that involve $\mathrm{CO}^{1}$ white dwarfs (WDs), whereas dust formation rarely occurs in "ONe novae" involving ONe WDs [20]. Emissions from $\mathrm{CO}$ molecules have been observed in several novae, usually as the vibrational first-overtone band around $2.3 \mu \mathrm{m}$. Such CO-forming novae generally produce dust grains in their late phases (e.g., [8] and references therein). It has therefore been thought that the formation of $\mathrm{CO}$ and other molecular species is associated with dust formation in novae [20], even though some novae have produced dust grains without detectable CO, e.g., V1280 Sco [7] and V339 Del [53].

In this review, we summarize the temporal evolution of the photometric and spectroscopic behavior of V2676 Oph and discuss the physical evolution of the nova, focusing in particular on molecule formation during the maximum phase. Finally we also discuss the mass of the WD component of V2676 Oph, and we summarize the lessons learned from this nova.

\section{Overview of V2676 Oph}

Nova V2676 Oph was discovered on UT 2012 March 25.8 (day $t=0$ ) by Japanese amateur astronomer H. Nishimura [40]. Immediately after the discovery the nova was confirmed as an "Fe II type" nova, according to the classification scheme of Williams [67], based on low-resolution spectroscopic observations [3]. The nova showed narrow emission lines of $\mathrm{H} \alpha, \mathrm{H} \beta, \mathrm{Fe}$ II, and $\mathrm{O}$ I, with P-Cygni profiles. Rudy et al. in 2012 [50] reported that the near-infrared spectra (from 0.8 to $2.4 \mu \mathrm{m}$ ) taken on UT 2012 March 28 and 30 showed Fe II emission lines together with low-excitation emission lines such as $\mathrm{C} \mathrm{I}, \mathrm{N} \mathrm{I}, \mathrm{O} \mathrm{I}$, and $\mathrm{Ca}$ II, in addition to $\mathrm{H}$ I emission lines. Many of the H I, O I, and N I emission lines had P-Cygni profiles. These observations confirmed that the nova was an Fe II type nova. However, there was no report of $\mathrm{CO}$ molecular-emission bands on those dates (cf., [51]). Although X-ray and UV observations were performed by the Swift satellite during the declining phase of the nova, no X-ray source was detected at its position, and only $U$-band magnitudes of the nova were measured [38,39]. Attempts to detect the nova in the radio domain also failed [39].

\footnotetext{
${ }^{1}$ Note that "CO" here refers to elemental carbon and oxygen abundances and not to the carbon monoxide molecule.
} 
As shown in Figure 1, the light curves of the nova in the optical and near-infrared-wavelength regions are characterized by (1) a slow evolution in brightness and (2) dust formation around day $t=90$. The early rate of decline of the brightness between 10 and 80 days after discovery was $\sim 0.01 \mathrm{mag} \mathrm{day}^{-1}$. The $V$-band slope of $0.013 \pm 0.004 \mathrm{mag} \mathrm{day}^{-1}$ during the early-decline phase indicates that V2676 Oph can be classified to the "slow" or "very slow" speed class [64]. The rates of decline for the other photometric bands $(B, V, R, I, J, H$, and $K)$ are listed in Table 1.

We note that V2676 Oph is similar to the dust-forming nova DQ Her that erupted in 1934 [35], based on the slow evolution of its brightness and the dust formation at day $t \sim 100$, in addition to the molecule formation just after the visual-brightness maximum (the $\mathrm{CN}$ molecule was detected in both novae). Based on the "nova wind" theory [24], the similar rates of decline in the brightness of V2676 Oph and DQ Her indicate that these novae involve WDs with similar masses. The mass of the WD component of DQ Her has been determined to be $(0.60 \pm 0.07) \mathrm{M}_{\odot}$ [26]. Therefore, V2676 Oph also probably involves a low-mass WD component, probably a CO WD of mass $\sim 0.6$ $\mathrm{M}_{\odot}$. The typical mass of a CO WD is smaller than that of an ONe WD (their masses may be distinguished by $\sim 1.1 \mathrm{M}_{\odot}$ [22]). No other parameters have ever been reported for the binary system hosting V2676 Oph (e.g., the masses of the WD and secondary star, the spectral type of the secondary star, the orbital period, and inclination angle of the binary system. The nature of the WD component of V2676 Oph is discussed further in the following sections.

The distance to V2676 Oph was determined by Raj et al. in 2017 [46] to be $d=6.9-7.9 \mathrm{kpc}$ (near the Galactic center), based on the absolute magnitude in the $V$-band at the visual-brightness maximum $\left(M_{V, \max }=-6.5\right.$ to -6.8 , as estimated from the Maximum Magnitude Rate of Decline (MMRD) relation [12] with $t_{2}=60-80$ days) and a visual extinction $A_{V}=2.9 \pm 0.1$, together with the apparent magnitude at the visual-brightness maximum $\left(m_{V}=10.6 \mathrm{mag}\right)$. Raj et al. derived the visual extinction by using the "Balmer decrement" method and their spectroscopic observations. It is noted that there are two issues to be re-examined. First, the MMRD relation used in their study may be inaccucate as pointed out some studies [42, 55, 24]. In addition to the MMRD relation, the visual extinction determined by the "Balmer decrement" method (which requires the assumptions about recombination of hydrogen atoms and physical conditions) may be not sufficiently accurate. Nagashima et al. in 2015 [37] had obtained $A_{V}=2.35 \pm 0.11$, from their spectroscopic observations, using the same method. Another determination based on a different method, by Kawakita et al. in 2016 [33], found $A_{V}=2.65 \pm 0.15$ by comparing the optical spectrum just after the visual-brightness maximum (which is dominated by absorption) with the synthesized spectrum of a supergiant.

Based on the estimated distance to V2676 Oph, Raj et al. determined the total mass of hydrogen in the ejecta from V2676 Oph to be $(0.27-3.3) \times 10^{-4} d^{2} \mathrm{M}_{\odot}$ from their observations during the early-decline phase [46]. Furthermore, Raj et al. recently determined the ejected mass from V2676 Oph to be $\sim 1.4 \times 10^{-5} \mathrm{M}_{\odot}$ based on the nebular phase spectrum of the nova [47]. They also estimated the dust mass formed in V2676 Oph was $(2.1-2.7) \times 10^{-8} \mathrm{M}_{\odot}$ [47]. The masses of the ionized ejecta from typical CO novae are estimated to be in the range $(1-10) \times 10^{-5} \mathrm{M}_{\odot}$ while those of ONe novae are in the range $(1-4) \times 10^{-4} \mathrm{M}_{\odot}$ based on observational studies (although these facts are not fully understood from the theoretical point of view) [20]. V2676 Oph is considered as a $\mathrm{CO}$ nova from the viewpoint of ejected mass. 

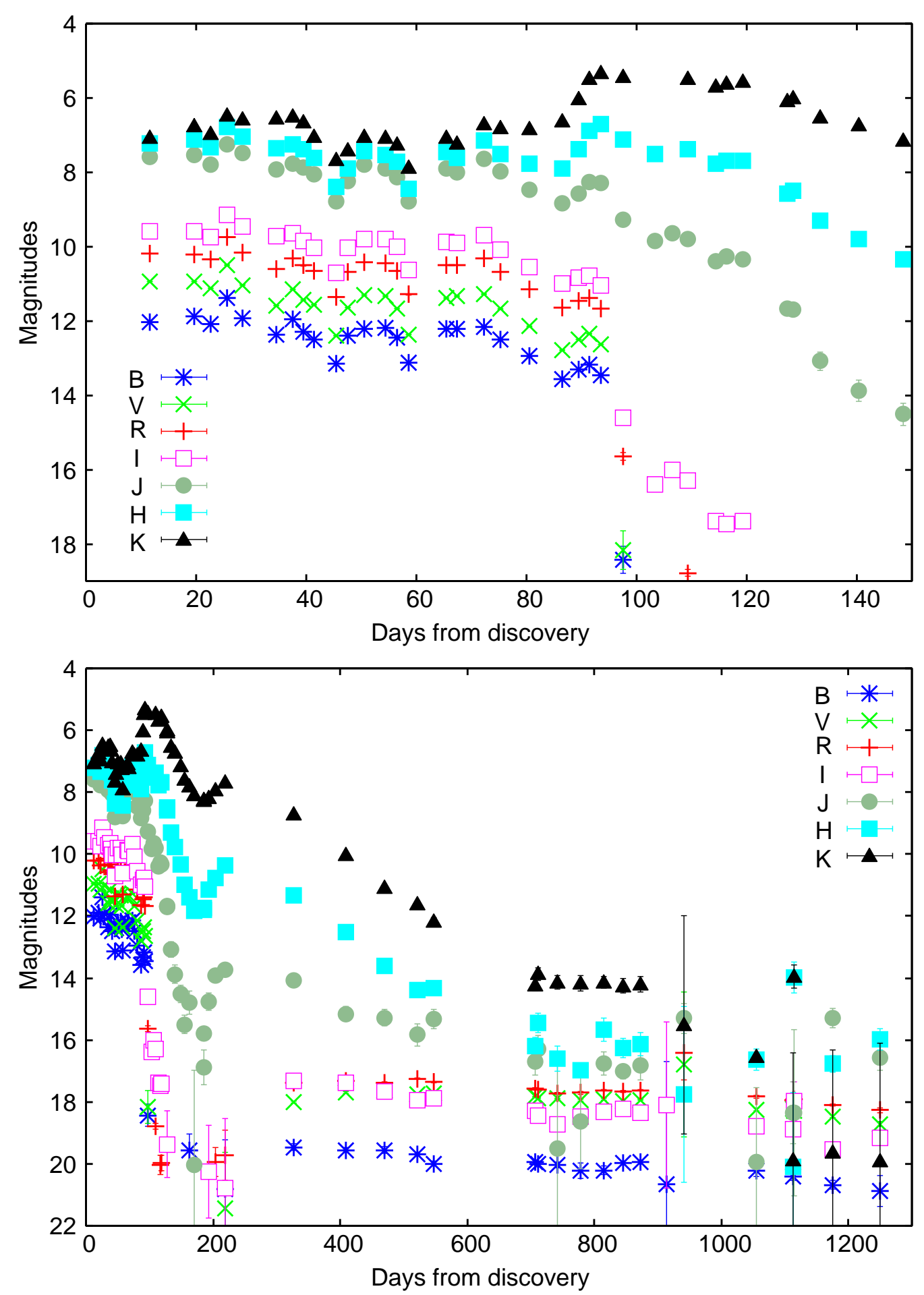

Figure 1: Multi-wavelength lightcurves (in the $B, V, R, I, J, H$, and $K$-bands) of V2676 Oph up to 150 days (upper panel) and up to 1300 days (lower panel) after its discovery. The photometric data are taken from SMARTS [63] (http://www.astro.sunysb.edu/fwalter/SMARTS/NovaAtlas/atlas.html). 


\begin{tabular}{cc}
\hline Photometric band & Decline rate $\left[\mathrm{mag} \mathrm{day}^{-1}\right.$ ] \\
\hline$B$ & $0.013 \pm 0.004$ \\
$V$ & $0.013 \pm 0.004$ \\
$R$ & $0.010 \pm 0.004$ \\
$I$ & $0.010 \pm 0.004$ \\
$J$ & $0.009 \pm 0.004$ \\
$H$ & $0.010 \pm 0.004$ \\
$K$ & $0.006 \pm 0.004$ \\
\hline
\end{tabular}

Table 1: Rates of decline of V2676 Oph in the optical and near-infrared bands during the early-decline phase (days $t=10-80$ ).

\section{Near Visual Maximum: Molecule Formation in V2676 Oph}

Low-resolution optical spectroscopic observations were performed by several observers around the visual-brightness maximum of V2676 Oph [36, 32, 46]. Just after its discovery, the emission lines were narrow, and the P-Cygni profile of $\mathrm{H} \alpha$ emission indicated a decrease in the expansion velocity of the nova outflow, from $\sim 750 \mathrm{~km} \mathrm{~s}^{-1}$ to $\sim 400 \mathrm{~km} \mathrm{~s}^{-1}$, during days $t=2-13$ [32]. The P-Cygni absorption profile of Na I indicated an outflow velocity of $600 \mathrm{~km} \mathrm{~s}^{-1}$ at day $t=10$ [46]. The emissions became progressively weaker until day $t=12$ (when almost no emission lines were seen in the observed spectrum). The visual maximum occurred at day $t \sim 10$. Near-infrared spectroscopic observations also reported weakened emission lines during this period [50]. After day $t=12$, the emission lines began to strengthen again (Figure 2).

These changes in the emission lines observed in V2676 Oph around its visual-brightness maximum can be explained by a model for slow novae proposed by Hachisu \& Kato in 2014 [23]. They hypothesized that the internal structure of a slow nova changes near its visual-brightness maximum, from a static configuration to a "nova wind" configuration. The optically thin wind first begins to blow during the static configuration just after the explosion, becoming weaker near the visual-brightness maximum. Finally, an optically "thick" wind starts to blow after the system enters a "nova wind" configuration. Note that such a static solution can be realized for WDs of mass $0.5-0.7 \mathrm{M}_{\odot}[23,31]$. It is therefore likely that V2676 Oph was powered by a thermonuclear runaway (TNR) on a WD of relatively small mass, as already discussed in Section 2.

The $\mathrm{C}_{2}$ Swan-band absorption and the $\mathrm{CN}$ "red system" band absorption were detected simultaneously at days $t=13$ and 14 [36, 32]. The $\mathrm{C}_{2}$ molecule has never been detected previously in other classical novae, whereas the CN molecule was detected clearly in DQ Her just after its visualbrightness maximum, and absorption due to both the $\mathrm{CN}$ violet- and red-system bands lasted about one week $[69,54,58,6,2,56]$. In the case of V2676 Oph, $\mathrm{C}_{2}$ and $\mathrm{CN}$ were detected on two nights only, but they may have been present for as long as one week. We did not find any other spectroscopic observations for days $t=15-19$. The $\mathrm{CN}$ violet-system absorption may also have been detected (but not well-confirmed) in other novae, e.g., in GK Per [70] and V445 Pup [27]. Figure 3 shows the spectrum at day $t=14$, together with the spectrum of a typical carbon star (TX Psc). Figure 4 shows the spectra at days $t=13$ and 14, together with a modeled spectrum of $C_{2}$. The excitation temperatures on those dates were $4500-5000 \mathrm{~K}$. Those values are lower than the 

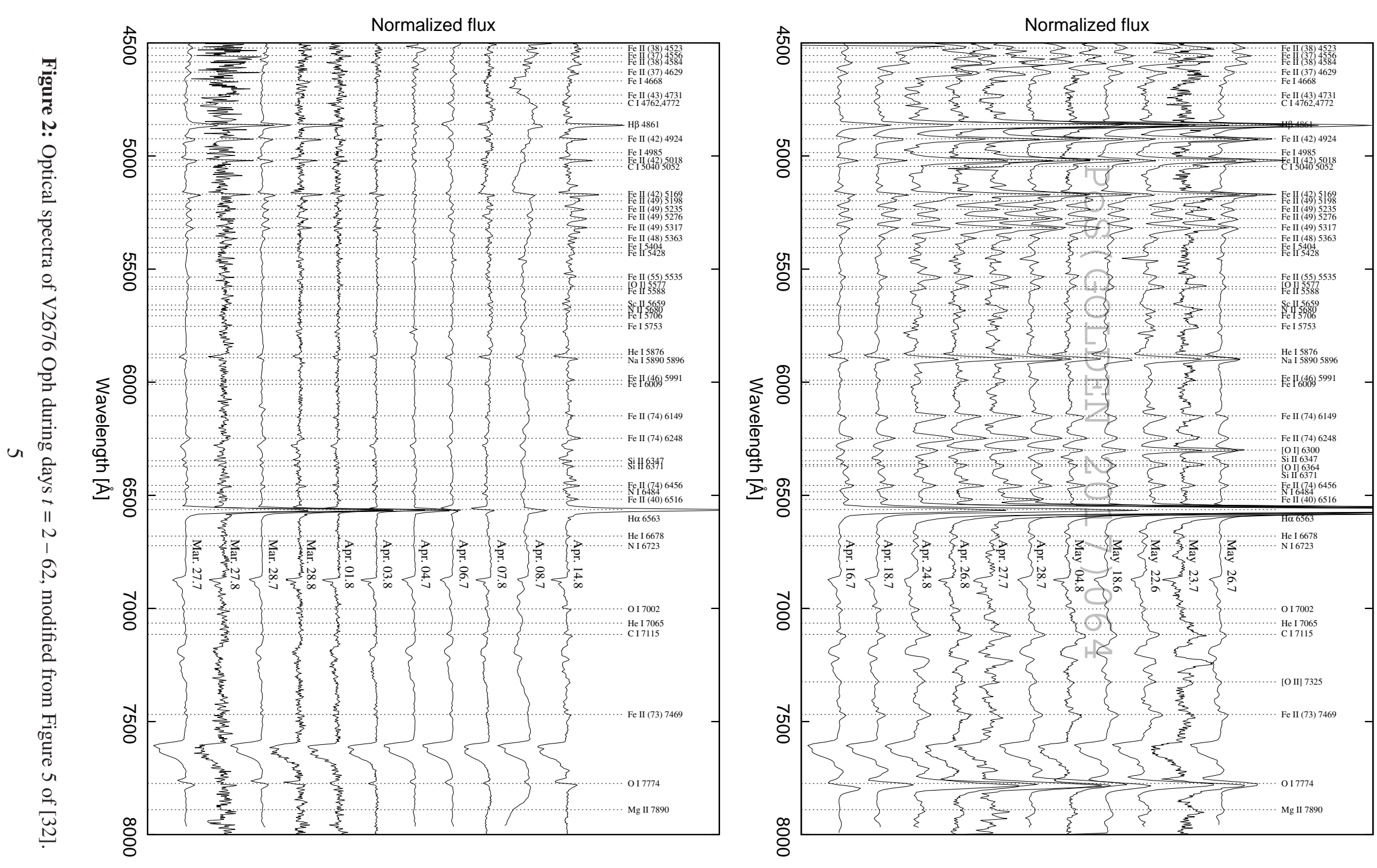
typical temperature of nova photosphere around the visual-brightness maximum, $\sim 8000 \mathrm{~K}[16]$. V2676 Oph was thus cool enough to form diatomic molecules near the visual maximum $\left(\mathrm{C}_{2}\right.$ and $\mathrm{CN}$ molecules can form at temperatures below $\sim 5000 \mathrm{~K}$ ). Furthermore, the strong $\mathrm{C}_{2}$ absorption bands that have never been observed previously in novae, and which are comparable to the $\mathrm{CN}$ absorption bands, probably indicate that the nova envelope gas was carbon-rich $(\mathrm{C} / \mathrm{O}>1)[43]$.

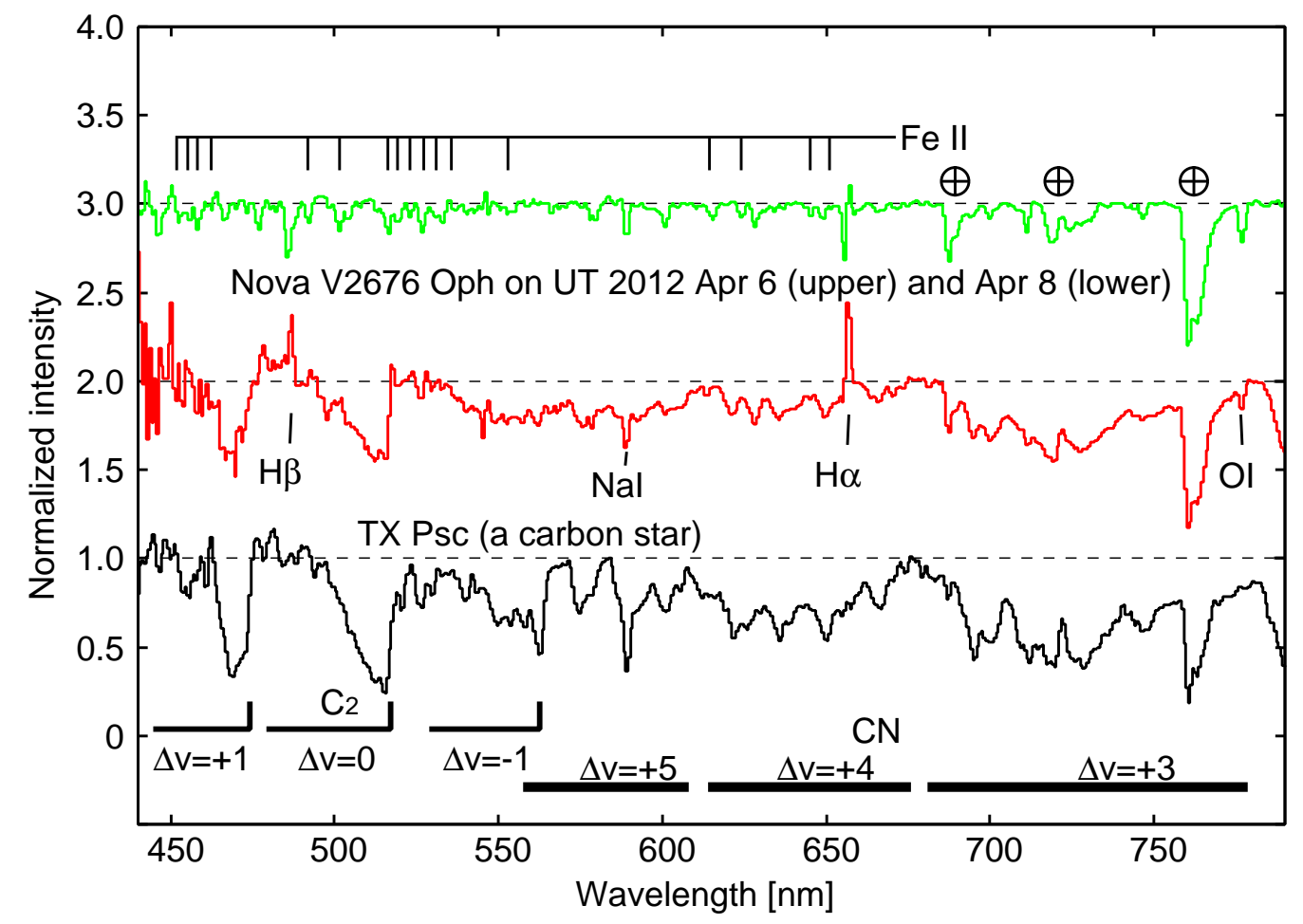

Figure 3: Normalized spectra of V2676 Oph on UT 2012 Apr 6 and 8 (days $t=12$ and 14, respectively) and of a carbon star (TX Psc), modified from Figure 3 of [36].

What was going on during the near-maximum phase that led to molecule formation? Where did the molecules form in the nova envelope: near the photosphere or in the outer envelope? Kawakita et al. in 2016 [33] investigated the evolution of the photospheric temperature of V2676 Oph based on the $(V-I)$ color index determined from their photometric observations. After correcting for interstellar reddening, they obtained the photospheric temperatures by assuming a hydrostatic stellar photosphere appropriate for a supergiant. The assumption of a hydrostatic atmosphere is not too bad because the evolution of V2676 Oph was very slow during the near-maximum phase (e.g., as is the case for a Cepheid variable)[33]. The photospheric temperatures were about $7000 \mathrm{~K}$ until the visual maximum at day $t \sim 10$ (Figure 5). As already noted in the previous section, the brightness changed slowly up to the time of visual maximum. For example, the $V$-band magnitudes were almost constant during days $t=3-8(V \sim 12 \mathrm{mag})$, and the brightness reached the maximum at day $t \sim 10$ after brightening by $\Delta m_{V} \sim 1.5 \mathrm{mag}$. There was no significant change in the photospheric temperature around the maximum. These facts imply that the photosphere had expanded radially without a significant increase in the photospheric temperature, indicating an increase in the luminosity of V2676 Oph. The energy source for this brightening may have been 


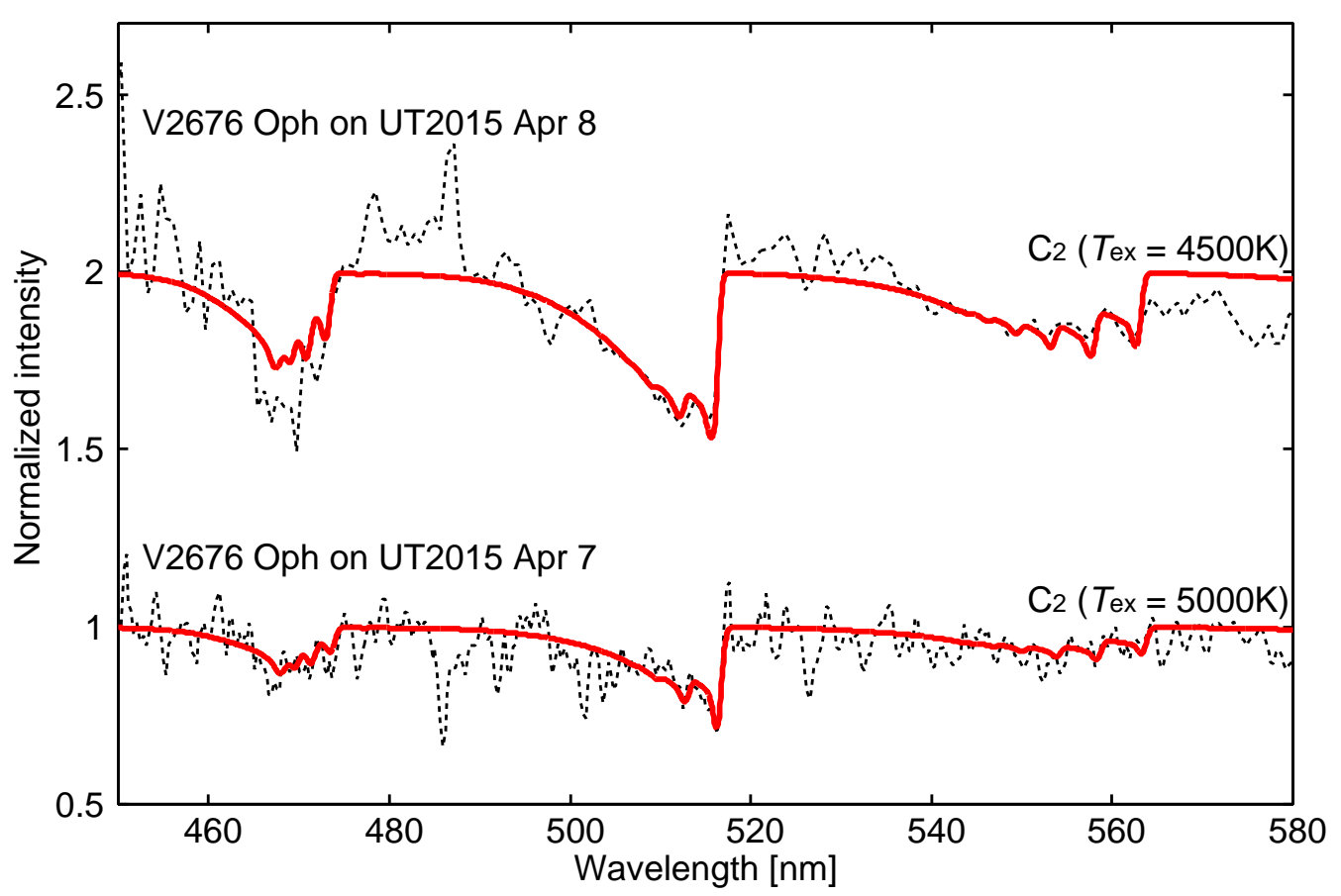

Figure 4: The Swan bands of $\mathrm{C}_{2}$ in V2676 Oph, modified from Figure 7 of [32]. The dotted lines are the observed spectra, and the solid lines are the modeled spectra of $\mathrm{C}_{2}$. The $C_{2}$ excitation temperatures were $\sim 5000 \mathrm{~K}$ and $\sim 4500 \mathrm{~K}$ on days $t=13$ and 14 , respectively.

supplied by a transition in the internal structure of the nova from a static configuration to a "nova wind" configuration, as noted by Kato \& Hachisu in 2009 [31]. At the same time, there were a few weak emission lines, and the expansion velocity was still as slow as $\sim 450 \mathrm{~km} \mathrm{~s}^{-1}$ [32], indicative of slowly expanding gas around the nova photosphere. If the optically thin wind had already ceased by that time, the observed emission lines may have arisen from remnants of gas ejected as an optically thin wind during an earlier phase.

The photospheric temperature of V2676 Oph decreased after the visual brightness maximum, up to day $t=14$, when the molecular formation of $\mathrm{C}_{2}$ and $\mathrm{CN}$ began. The cooling timescale $(e-$ folding time) during this period was $\sim 9$ days [33], which is comparable to that due to radiative cooling by $\mathrm{CO}$ molecules [14]. Because the binding energy of the $\mathrm{CO}$ molecule is larger than that of $\mathrm{C}_{2}$ or $\mathrm{CN}$ (see Table 2), the $\mathrm{CO}$ molecules may have formed earlier, before the formation of the $\mathrm{C}_{2}$ and $\mathrm{CN}$ molecules. The UV continuum absorption by neutral atoms such as $\mathrm{C} \mathrm{I}$ and $\mathrm{Fe} \mathrm{I}$ (which was seen in the optical spectra as line absorption [33]) probably prevented the hard radiation from the nova photosphere. This may have promoted the formation of those diatomic molecules. The CO molecule was successfully detected in V2676 Oph, but only during the early-decline phase (days $t=37$ and 38) [51]. Unfortunately, no spectroscopic observations of CO molecules during the near-maximum phase have been reported so far.

Based on their model atmospheres, Hauschildt et al. in 1994 [25] discussed the formation of $\mathrm{CN}$ in nova atmospheres theoretically, especially for the case of DQ Her. They pointed out that nonLTE effects in nova atmospheres are large and very important due to the large radial extension of and the huge temperature gradient in — the nova photosphere. Their models (with enhanced CNO 
abundances relative to the solar abundances) reproduced not only the $\mathrm{CN}$ absorption bands in the optical wavelength region but also the infrared $\mathrm{CO}$ emission bands. The electron temperatures may be low $(\sim 4000 \mathrm{~K})$ in the line-forming region of the nova atmosphere, and such low temperatures are consistent with the excitation temperatures of $\mathrm{CN}$ and $\mathrm{C}_{2}$ in novae [56, 32], as well as those of the $\mathrm{CO}$ molecule (e.g., [8]).

Finally, we note that - in novae - the molecular bands of both $\mathrm{CN}$ and $\mathrm{C}_{2}$ have been observed in absorption, while the vibrational bands of the $\mathrm{CO}$ molecule have always been observed in emission. Because the excitation temperatures of $\mathrm{C}_{2}$ (and probably $\mathrm{CN}$ ) in V2676 Oph are comparable to the effective temperature of the nova photosphere at the epoch of molecule formation (Figure 5), it is likely that both $\mathrm{CN}$ and $\mathrm{C}_{2}$ in V2676 Oph were concentrated near the photosphere when it was cool enough for those molecules to form $(<\sim 5000 \mathrm{~K})$. On the other hand, the CO vibrational bands were observed in emission for V2676 Oph during its early-decline phase. This fact indicates that the distribution of $\mathrm{CO}$ molecules extended beyond the nova photosphere at that time. Even during the near-maximum phase, the vibrational band of $\mathrm{CO}$ has been detected in emission in some novae (e.g., V705 Cas [14]), and it probably already extended beyond the photosphere at that time. If the $\mathrm{CO}$ molecules were collisionally excited, radiative cooling by the $\mathrm{CO}$ molecules would have cooled down the outer envelope. Indeed, the excitation temperatures of $\mathrm{CO}$ molecules observed in novae are in the range of $2500-4500 \mathrm{~K}$, significantly lower than the typical photospheric temperatures of novae ( $>\sim 8000 \mathrm{~K})$. Otherwise, if the $\mathrm{CO}$ molecules were mainly excited by radiation from the central nova, the relatively lower excitation temperatures of the $\mathrm{CO}$ molecules in novae may be explained by dilution of the radiation field.

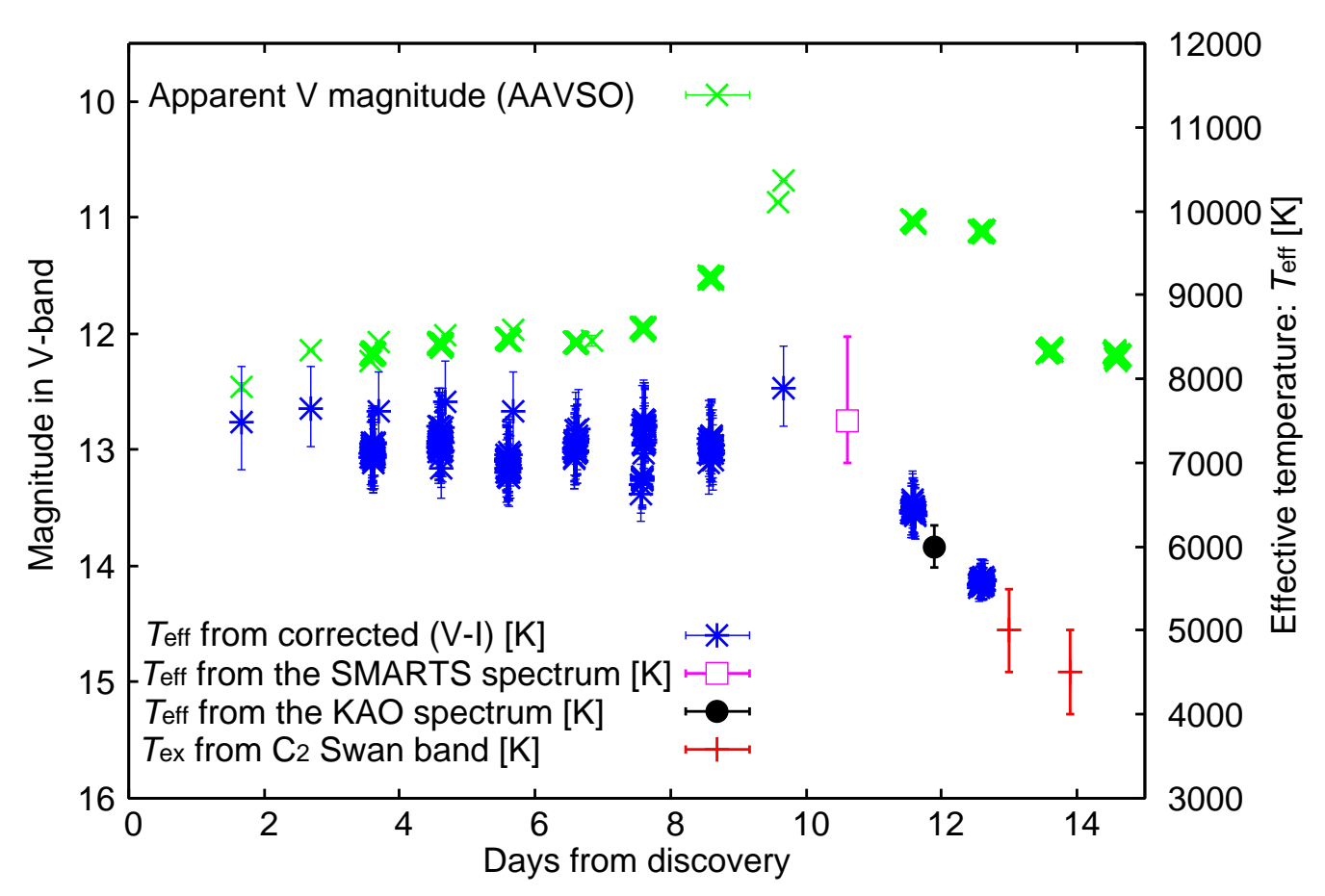

Figure 5: Effective temperatures of the nova photosphere of V2676 Oph during its near-maximum phase, modified from Figure 5 of [33]. 


\begin{tabular}{lll}
\hline Atom & $\begin{array}{l}\text { Ionization } \\
\text { potential }[\mathrm{eV}]\end{array}$ & $\begin{array}{l}\text { Equivalent } \\
\text { wavelength [nm] }\end{array}$ \\
\hline $\mathrm{H} \mathrm{I}$ & 13.6 & 91.2 \\
$\mathrm{He} \mathrm{I}$ & 24.6 & 50.4 \\
$\mathrm{C} \mathrm{I}$ & 11.3 & 110.1 \\
$\mathrm{~N} \mathrm{I}$ & 14.5 & 85.3 \\
$\mathrm{O} \mathrm{I}$ & 13.6 & 91.0 \\
Fe I & 7.90 & 156.9 \\
\hline \hline Molecule & Dissociation & Equivalent \\
& energy [eV] & wavelength [nm] \\
\hline $\mathrm{CO}$ & 11.1 & 111.8 \\
$\mathrm{C}_{2}$ & 8.6 & 143.6 \\
$\mathrm{CN}$ & 7.7 & 160.0 \\
$\mathrm{H}_{2}$ & 4.5 & 276.9 \\
\hline
\end{tabular}

Table 2: Wavelength limits for continuum absorption by neutral atoms and the wavelengths necessary for photodissociation of selected molecules (after Table 2 of [33]).

\section{Early-Decline Phase}

Raj et al. in 2017 [46] pointed out that the $V$-band lightcurve exhibited fluctuations of $\sim 0.7$ mag around $V=11.5$ until about 70 days after the outburst, before beginning a slow decline (from days $\sim 70$ to $\sim 90$ ), as shown in Figure 1. Such re-brightening events may be associated with the re-appearance of P-Cygni profiles, as can be seen by comparing the optical spectra [46] with the optical lightcurves. Such re-appearance of the P-Cygni profiles could be interpreted as a reexpansion of the nova photosphere $[60,61]$. However, we have to examine this hypothesis in future studies based on a sophisticated physical model of expanding nova envelope.

During the early-decline phase, the expansion velocity of V2676 Oph increased. The $\mathrm{H} \alpha$ emission line was systematically broader after day $t=32$, whereas the line profile was narrower until at least day $t=20$ [32]. The P-Cygni profile of $\mathrm{Na}$ I showed an absorption component at -600 $\mathrm{km} \mathrm{s}^{-1}$ (day $\left.t=22\right)$ and $-660 \mathrm{~km} \mathrm{~s}^{-1}$ (day $t=26$ ) [46]. After that, an absorption component was observed at $-950 \mathrm{~km} \mathrm{~s}^{-1}$ in the P-Cygni profiles of Fe II on day $t=34$. The absorption component of the P-Cygni profiles of the Balmer emission lines accelerated to a velocity of about $-1000 \mathrm{~km}$ $\mathrm{s}^{-1}$ (day $t=67$ ) and then to about $-1100 \mathrm{~km} \mathrm{~s}^{-1}$ (day $t=91$ ) [46]. Figure 6 shows the change in the expansion velocities of the nova outflow in V2676 Oph. The expansion velocities decreased from the pre-maximum to the maximum phase, and then increased again during the early-decline phase. Such changes in the absorption components of the P-Cygni profiles were also observed in DQ Her $[1,35]$, which is one of the slow novae. It also showed CN absorption, as mentioned in Section 2. V2676 Oph and DQ Her are quite similar to each other in those respects. Furthermore, such acceleration of the absorption lines during the early-decline phase has sometimes been recognized in high-resolution spectra of novae (e.g., [68, 4]).

As noted in the previous section, Rudy et al. in 2012 [51] detected CO molecules in V2676 
Oph during the early-decline phase (days $t=37$ and 38). They reported the detection of CO emission from the fundamental and the first and second overtones in V2676 Oph. The fundamental band is much weaker than the first-overtone band, indicating that the $\mathrm{CO}$ emission was optically thick in the fundamental band. The peak of the $\mathrm{CO}$ first-overtone band is approximately seven times stronger than the underlying continuum (this is the highest ratio ever observed in a nova [51]). The strong $\mathrm{CO}$ emission indicates the release of considerable energy from the expanding nova envelope, which promotes the cooling of the gas and results in dust formation. The presence of $\mathrm{CO}$ emission in the $K$-band (the first-overtone band of $\mathrm{CO}$ is at $\sim 2.3 \mu \mathrm{m}$ ) may explain the relatively shallower slope of the $K$-band lightcurve for days $t=10-80$ (see Table 1 ).

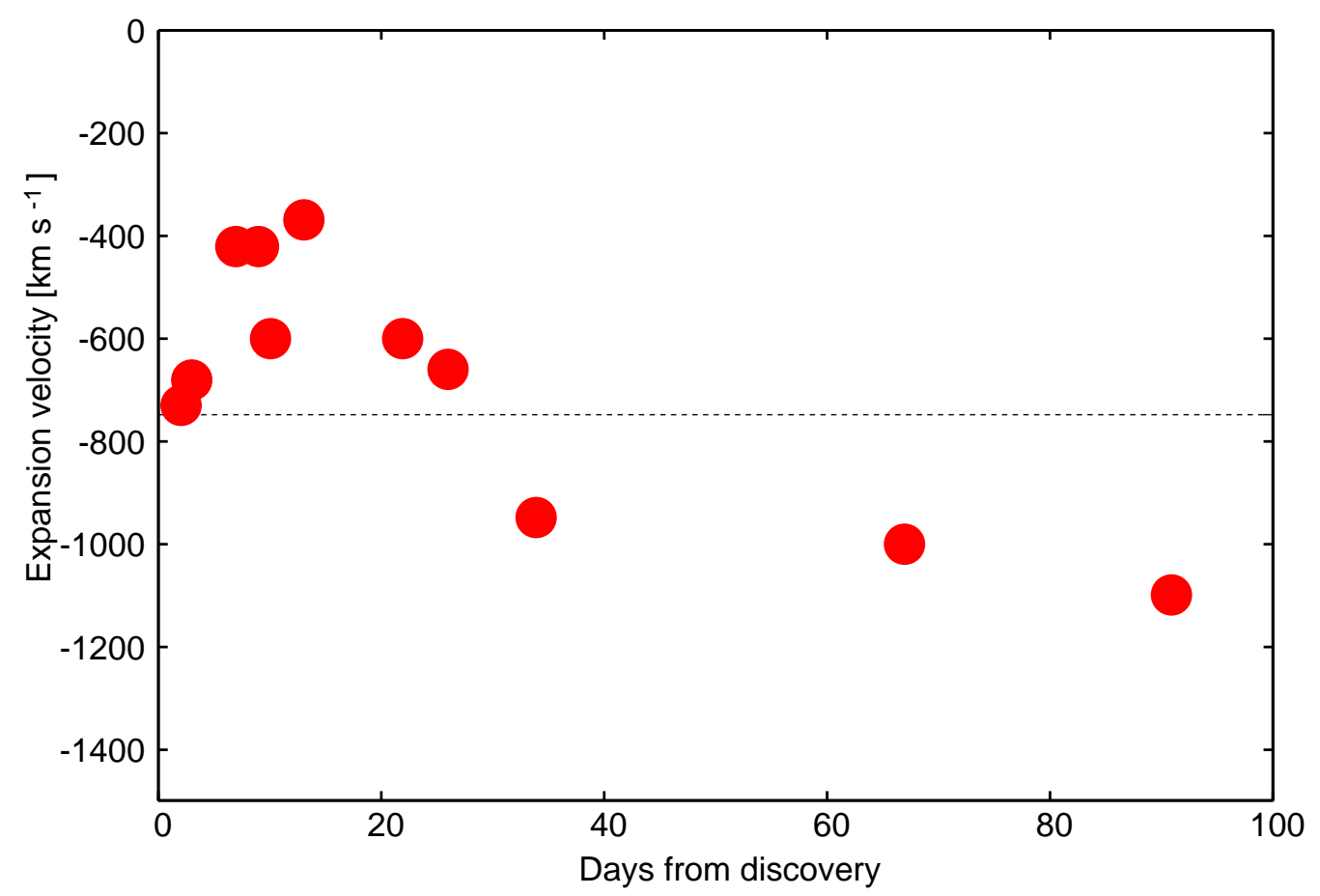

Figure 6: Expansion velocities of the nova outflow observed in V2676 Oph $[32,46]$. The expansion velocity decelerated during the pre-maximum phase, while it accelerated after the visual-brightness maximum (day $t \sim 10$ ). The horizontal dotted line corresponds to $-750 \mathrm{~km} \mathrm{~s}^{-1}$ (corresponding to the maximum expansion velocity obtained from the emission lines during the nebular phase; see Section 6).

\section{Dust Formation}

The near-infrared brightness (in the $H$ and $K$ bands) of V2676 Oph increased suddenly at day $t \sim 90$ [46]. At the same time, the visual lightcurves started to decrease rapidly in brightness. Both behaviors in the lightcurves are associated with dust formation, which results in thermal emission from dust grains at near-infrared wavelengths and visual extinction by dust grains at optical wavelengths. On day $t=93$ (at the peak of the $K$-band brightness), the color index $(J-K)$ was about -3.1 , corresponding to a blackbody temperature of $1500-2000 \mathrm{~K}$ [46]. This temperature range is consistent with dust condensation in the nova outflow around day $t=90$. Furthermore, photometry 
in the near-infrared wavelength region between days $t=164$ and 380, which was reported in [62], also confirms the presence of dust grains.

Direct evidence for the existence of dust grains was obtained by mid-infrared spectroscopic and photometric observations of V2676 Oph, carried out after dust formation, at days $t=452$ and 782 [34]. Both amorphous carbon grains and silicate grains were found to exist simultaneously in V2676 Oph (Figure 7). According to the traditional paradigm of CO saturation for grain formation, only carbon-rich grains form in gas with $\mathrm{C} / \mathrm{O}>1$ (all oxygen atoms being exhausted by the formation of $\mathrm{CO}$ molecules), and only silicate grains (containing oxygen atoms) form when $\mathrm{C} / \mathrm{O}<$ 1 (all carbon atoms being fixed in CO molecules). The existence of both types of grains in V2676 Oph indicates non-thermal-equilibrium conditions for the dust formation [20].

The unidentified-IR (UIR) emission line at $11.4 \mu \mathrm{m}$ which is sometimes observed in dustforming novae (e.g., V705 Cas [15]), was also detected in V2676 Oph [34]. This emission may be due to free-flying PAH molecules or HAC grains. Because free-flying PAH molecules cannot survive in the strong radiation field from the central nova during its later phase, the observed UIR feature may be due to HAC grains [13]. Kawakita et al. in 2017 [34] also reported the absence of strong [Ne II] emission at $12.8 \mu \mathrm{m}$, which is usually identified in the late phase of ONe novae. This fact strongly supports the conclusion that V2676 Oph involves a CO WD.

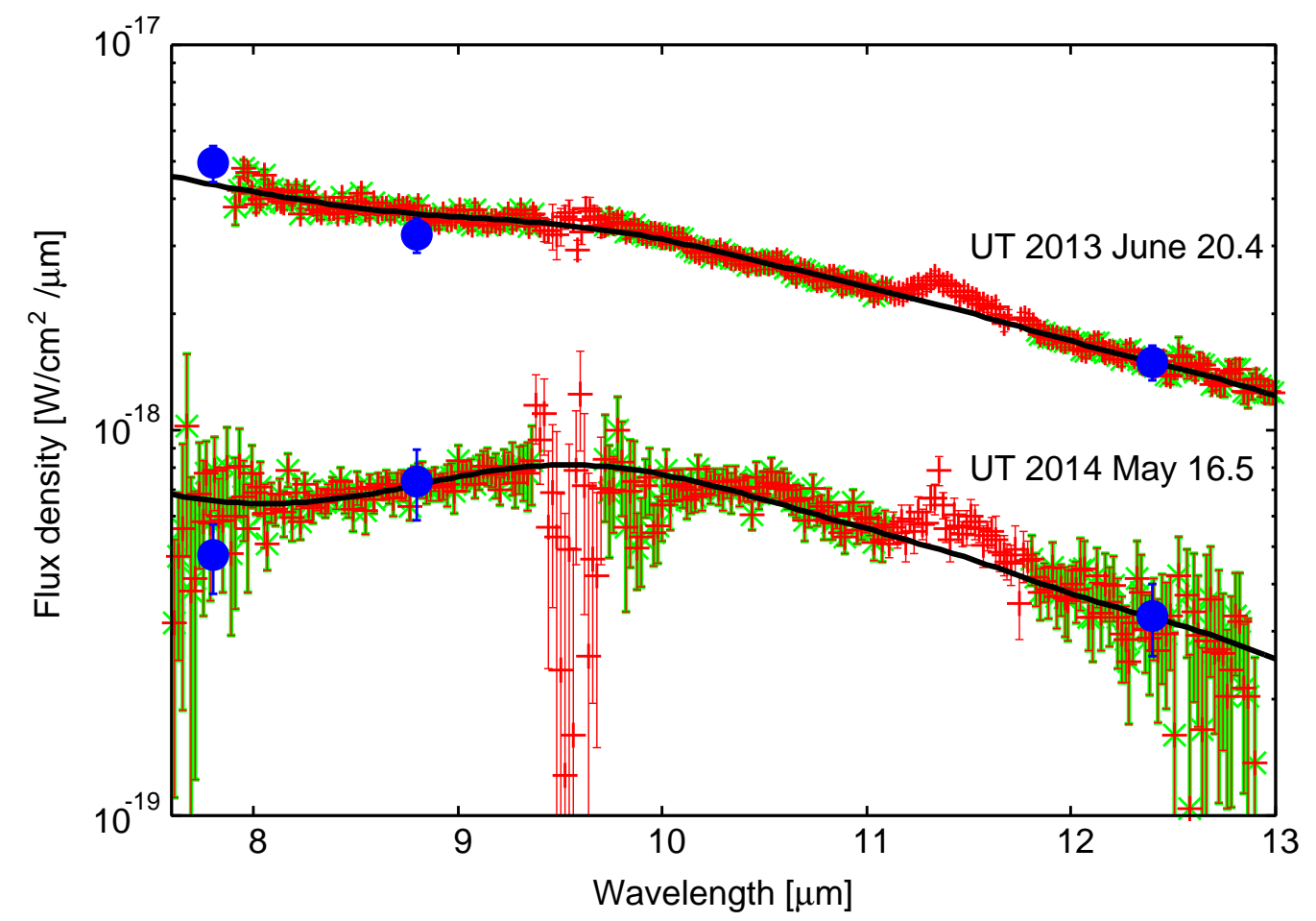

Figure 7: Mid-infrared low-resolution spectra of V2676 Oph at days $t=452$ and 782, modified from Figure 1 of [34]. The black solid lines represent the synthesized spectra at each epoch. The spectroscopic data used for the fit are shown in green; the wavelength regions contaminated by telluric ozone absorption around 9.6 $\mu \mathrm{m}$ and by UIR emission at $11.4 \mu \mathrm{m}$ are eliminated for the fit. The filled blue circles are the photometric measurements. 


\section{Nebular Phase}

In the nebular phase of a nova (during which the optically thin emission lines are observed), the shapes of some emission lines provide useful information about the geometry of the nova ejecta (e.g., [21]). Raj et al. in 2017 [46] reported a medium-resolution spectrum of V2676 Oph at day $t$ $=1138$, for which the instrumental resolution was about $100 \mathrm{~km} \mathrm{~s}^{-1}$. Figure 8 shows the emissionline profiles of [N II] (at 654.8 and $658.3 \mathrm{~nm}$, associated with the same upper energy level). Since parts of those emission lines overlap with the $\mathrm{H} \alpha$ emission line at $656.3 \mathrm{~nm}$, we extracted the blue-side profile of the [N II] at $654.8 \mathrm{~nm}$ and the red-side profile of the [N II] at $658.3 \mathrm{~nm}$. The intensity ratio of those two [N II] lines is consistent with the ratio of Einstein's $A$ coefficients for those transitions $(\sim 3)$. We fitted the profile with three Gaussian components. As shown in Figure 8 , the resultant components are centered at $213 \mathrm{~km} \mathrm{~s}^{-1},-21 \mathrm{~km} \mathrm{~s}^{-1}$, and $-185 \mathrm{~km} \mathrm{~s}^{-1}$, with FWHMs of $302 \mathrm{~km} \mathrm{~s}^{-1}, 149 \mathrm{~km} \mathrm{~s}^{-1}$, and $367 \mathrm{~km} \mathrm{~s}^{-1}$, respectively.

The double-peaked profiles seen in the [N II] emission lines are also recognized in other weak emission lines (such as [O I] and [S III]) in the nebular-phase spectrum of V2676 Oph. Figure 9 compares the emission lines of [N II], [OI], and [S III]. In this figure, each profile is normalized at its peak (before normalization, the intensity ratios of the doublet of [O I] and [S III] are consistent with the corresponding ratios of Einstein's $A$ coefficients for the respective transitions). We also shifted the wavelengths of the [O I] and [S III] lines to align their red components (which have positive velocities) with the red peak of the [N II] line in the velocity axis of Figure 9. Although the $\mathrm{S} / \mathrm{N}$ ratios for the $[\mathrm{O} \mathrm{I}]$ and $[\mathrm{S} \mathrm{III]} \mathrm{lines} \mathrm{are} \mathrm{not} \mathrm{as} \mathrm{good} \mathrm{as} \mathrm{for} \mathrm{[N} \mathrm{II],} \mathrm{their} \mathrm{profiles} \mathrm{are} \mathrm{basically}$ consistent with one another. The Full Width at Zero Intensity (FWZI) of the lines is about \pm 750 $\mathrm{km} \mathrm{s}^{-1}$, and each emission profile could be fitted with a combination of two or three Gaussian components, at velocities of $\sim 0 \mathrm{~km} \mathrm{~s}^{-1}$ and $\pm \sim 200 \mathrm{~km} \mathrm{~s}^{-1}$. Although the emission lines of [S III] at $906.9 \mathrm{~nm}$ and $953.1 \mathrm{~nm}$ are associated with the same upper energy level, their emission-line profiles are slightly different for the blue components (peaked at about $-200 \mathrm{~km} \mathrm{~s}^{-1}$ ). The line profile of [S III] at $906.9 \mathrm{~nm}$ is similar to that of [N II], but the emission profile of [S III] at 953.1 $\mathrm{nm}$ may be affected by strong telluric absorption lines in this wavelength region. Alternatively, the blue component of the [S III] at $953.1 \mathrm{~nm}$ may be weaker than the red component of the same [S III] line due, e.g., to a difference in opacities.

Derdzinski et al. in 2017 [11] recently proposed that dust formation in novae occurs in the dense, cool environments created by radiative shocks. A dense and equatorially concentrated ring of ejecta from the nova explosion is followed by a faster and isotropic nova wind that collides with the slower ejecta, producing shocks in the equatorial plane. Such dense and cool environments are suitable for dust formation. Therefore, if our line-of-sight is close to the equatorial plane of the binary system associated with the nova, we expect to observe a deep drop in the optical lightcurves produced by the strong extinction, and simultaneously we expect an enhancement of the brightness in the infrared-wavelength region. This picture (see also Figure 1 of [11]) is consistent with the dust-forming nova DQ Her, in which the inclination angle of the related binary system is $86.5 \pm 1.6$ degrees, close to the line-of-sight [26]. The line profiles of V2676 Oph shown in Figures 8 and 9 are also consistent with an expanding ring of equatorially concentrated ejecta, viewed nearly edge-on (i.e., at an inclination angle of $\sim 90$ degrees for the binary system hosting the explosion of V2676 Oph). The two velocity components that peak at $\pm \sim 200 \mathrm{~km} \mathrm{~s}^{-1}$ thus originate from 


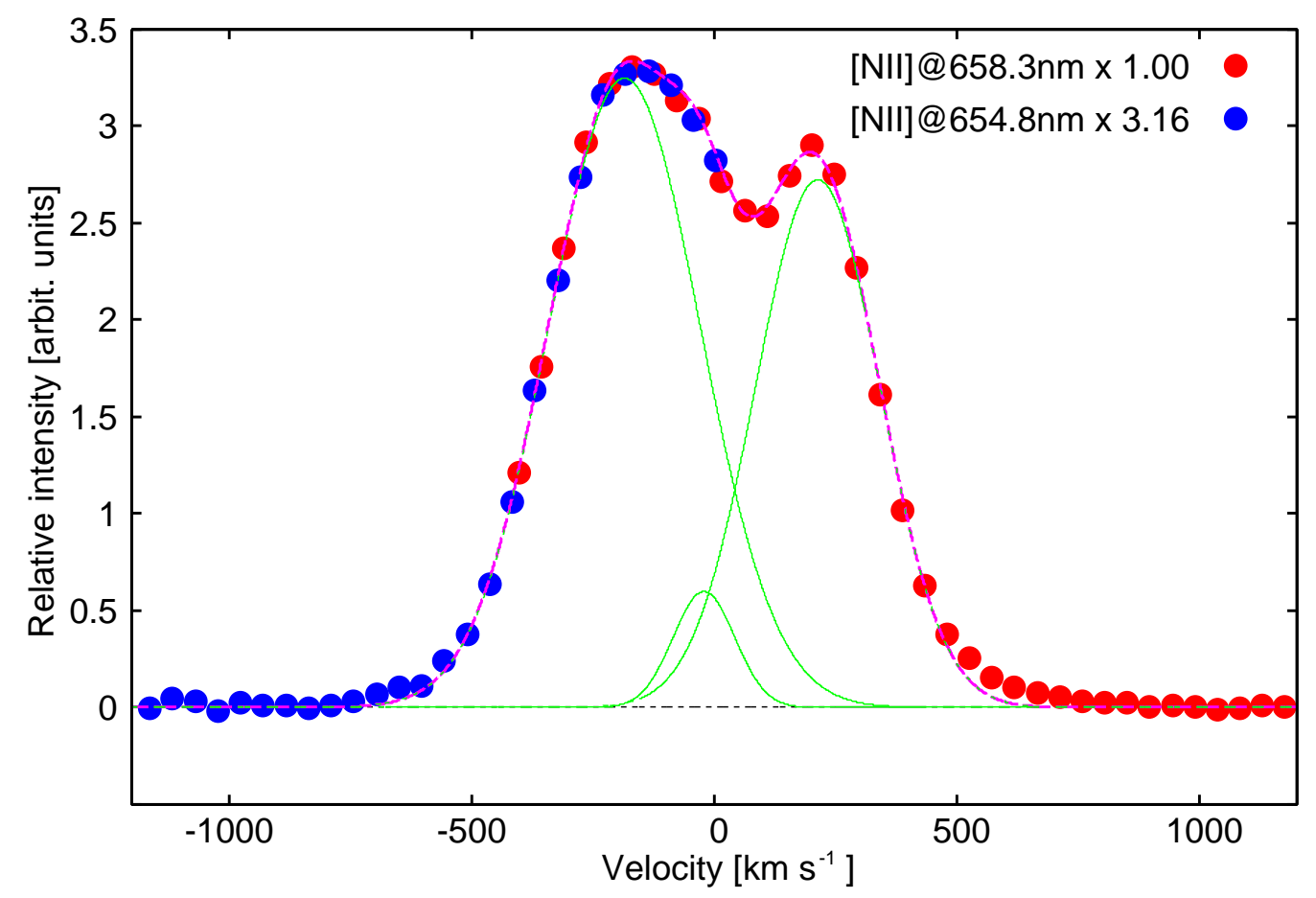

Figure 8: The [N II] emission lines of V2676 Oph during the nebular phase. At least two velocity components are prominent. The FWZI is about $\pm 750 \mathrm{~km} \mathrm{~s}^{-1}$. The original data was provided by Raj (2017, private communication) and processed by the authors, e.g., by the subtraction of a baseline, fitting with Gaussian profiles, etc. The solid and dashed lines are the best-fit Gaussian functions (see text).

the near-side and far-side ejecta (the velocity component at $\sim 0 \mathrm{~km} \mathrm{~s}^{-1}$ may correspond to gas moving in directions perpendicular to the equatorial plane, as in bipolar jets). The measured FWZIs of the forbidden emission lines are about $750 \mathrm{~km} \mathrm{~s}^{-1}$, comparable to the expansion velocities measured during the pre-maximum phase and the early-decline phase (Figure 6). The red (far-side) component of [N II] emission is slightly weaker than the blue (near-side) component, which may be caused by extinction produced by dust grains that remain in the emission region of the nova envelope.

\section{Lessons Learned from V2676 Oph: Is the WD mass small or large?}

The detection of both $\mathrm{C}_{2}$ and $\mathrm{CN}$ (along with CO) in V2676 Oph is interesting, because it indicates that the atmosphere of the nova envelope is carbon-rich $(\mathrm{C} / \mathrm{O}>1)$ [36], based on theoretical calculations of chemical evolution in nova outflows [43]. The enrichment of carbon relative to oxygen in V2676 Oph may have originated in mass transfer from a carbon star (as the secondary star) to the WD in the binary system associated with V2676 Oph. The elemental abundances of the nova envelope must also have been affected by the composition of the WD because matter accreted from the secondary star (even if it has solar-like abundances) mixes with the surface materials of the WD by diffusion before the TNR ignited on the WD. Traditional simulations of nova explosions based on TNR theory indicate that oxygen-rich ejecta are expected, rather than carbon-rich ejecta, 


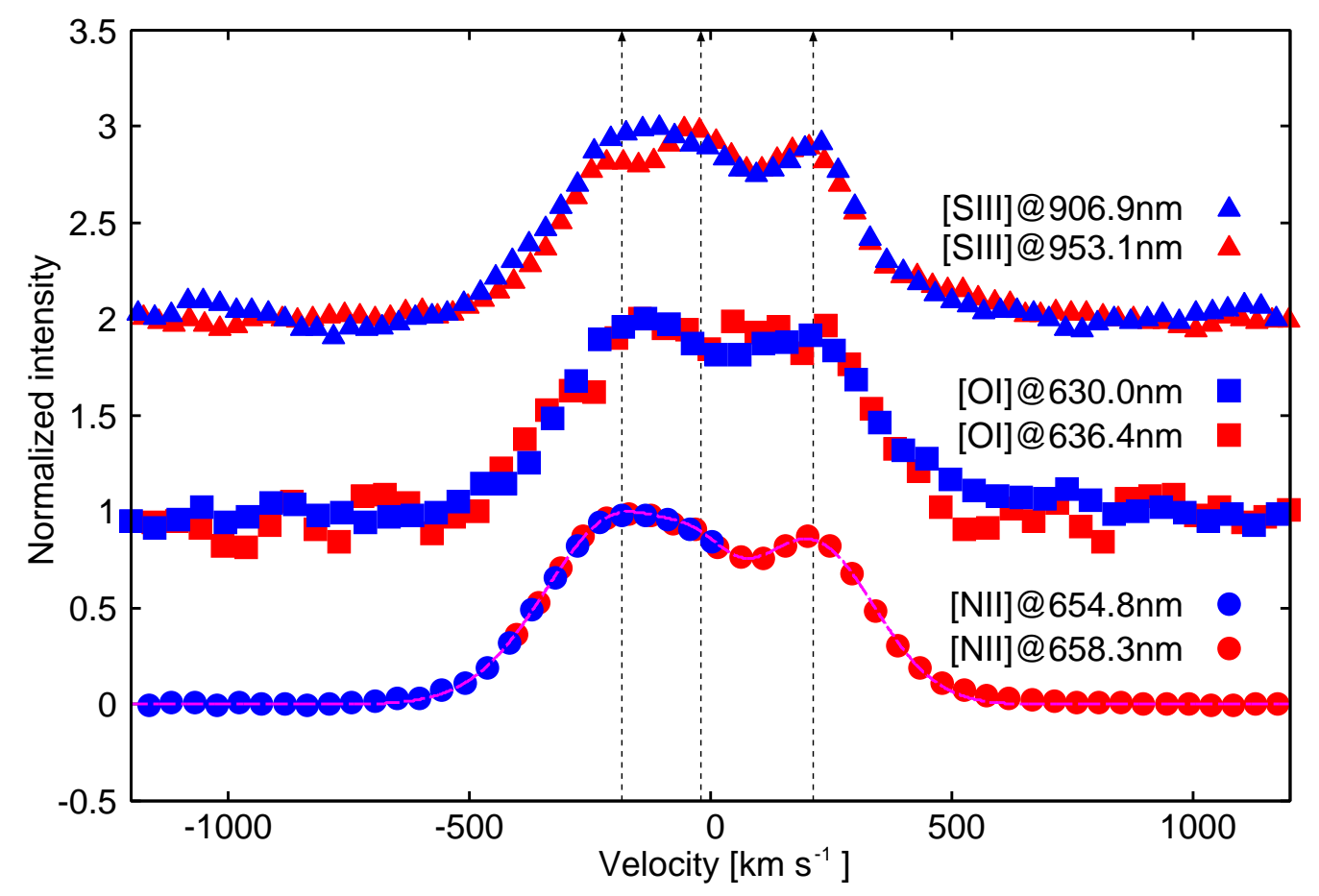

Figure 9: Comparison of the emission-line profiles of [N II], [O I], and [S III] (normalized at their peaks) during the nebular phase of V2676 Oph. The bottom profile is for [N II] (the same as in Figure 8, but normalized), the middle one is for [O I] (with an offset of 1.0) and the top profile is for [S III] (with an offset of 2.0). The vertical dashed lines indicate the velocities of the three Gaussian components fitted to [N II].

except for a few cases with heavy $\left(\sim 1.35 \mathrm{M}_{\odot}\right)$ ONe WDs (e.g., [28, 10]). However, prominent [Ne II] emission at $12.8 \mu \mathrm{m}$ was not observed in the mid-infrared spectra of V2676 Oph (see Section 5), indicating that V2676 Oph involves a CO WD. The estimated ejecta mass of V2676 Oph is also consistent with a CO nova (see Section 2). Furthermore, the estimated mass of the WD component may be as small as $\sim 0.6 \mathrm{M}_{\odot}$ (consistent with a $\mathrm{CO} \mathrm{WD}$ ), from the viewpoint of the photometric and spectroscopic evolution of the nova (see Sections 2 and 3). These discrepancies may be due to incorrect assumptions employed in the simulations of nova explosions. As pointed out by Starrfield in 2008 [57], the outer regions of the cores of CO WDs have $\mathrm{C} / \mathrm{O}$ ratios that differ strongly from unity, in contrast to the usual assumptions employed in traditional simulations $[5,22,10,30]$. Recent stellar-evolution models predict $\mathrm{C} / \mathrm{O}>1$ for the outer layers of CO WDs [30]. Therefore, the carbon-rich ejecta from V2676 Oph may originate from a CO WD, even if the secondary star has a solar-like composition. Accordingly, further theoretical studies of nova explosions (and of the prior stellar evolution leading to the formation of WDs) are to be encouraged.

Kawakita et al. in 2015 [32] have determined the isotopic ratios of carbon and nitrogen $\left({ }^{12} \mathrm{C} /{ }^{13} \mathrm{C} \sim 4\right.$ and $\left.{ }^{14} \mathrm{~N} /{ }^{15} \mathrm{~N} \sim 2\right)$ in V2676 Oph, based on the profiles of the molecular-absorption bands of $\mathrm{C}_{2}$ and $\mathrm{CN}$ observed in the nova. These observations determined for the first time the ${ }^{14} \mathrm{~N} /{ }^{15} \mathrm{~N}$ in a classical nova, while the ${ }^{12} \mathrm{C} /{ }^{13} \mathrm{C}$ ratio has been determined in several novae from observations of the $\mathrm{CO}$ first-overtone vibrational band (Table 3 ). The first simultaneous determination of the isotopic ratios of both carbon and nitrogen have allowed us to compare these ratios in nova 


\begin{tabular}{llll}
\hline Nova & ${ }^{12} \mathrm{C} /{ }^{13} \mathrm{C}$ & ${ }^{14} \mathrm{~N} /{ }^{15} \mathrm{~N}$ & Remarks \\
\hline DQ Her & $\geq 1.5$ & $\geq 2$ & $\mathrm{CN}[56]$ \\
NQ Vul & $>3$ & & $\mathrm{CO}[18]$ \\
V842 Cen & $2.9 \pm 0.4$ & & $\mathrm{CO}[65,66]$ \\
V705 Cas & $\geq 5$ & & $\mathrm{CO}[14]$ \\
V2274 Cyg & $1.2 \pm 0.3$ & $\mathrm{CO}[48]$ \\
V2615 Oph & $>2$ & $\mathrm{CO}[9]$ \\
V496 Sct & $\geq 1.5$ & & $\mathrm{CO}[44]$ \\
& $\sim 1.3$ & & $\mathrm{CO}[49,52]$ \\
V2676 Oph & $\sim 4$ & $\sim 2$ & $\mathrm{C}_{2}, \mathrm{CN}[32]$ \\
V5668 Sgr & $\sim 1.5$ & & $\mathrm{CO}[8]$ \\
\hline
\end{tabular}

Table 3: Summary of ${ }^{12} \mathrm{C} /{ }^{13} \mathrm{C}$ and ${ }^{14} \mathrm{~N} /{ }^{15} \mathrm{~N}$ ratios observed in novae.

ejecta with those of "pre-solar" grains found in meteorites [41]. It has been proposed theoretically that some pre-solar grains with low ${ }^{12} \mathrm{C} /{ }^{13} \mathrm{C}$ and low ${ }^{14} \mathrm{~N} /{ }^{15} \mathrm{~N}$ ratios may have originated in the TNR of novae [29]. The ${ }^{12} \mathrm{C} /{ }^{13} \mathrm{C}$ and ${ }^{14} \mathrm{~N} /{ }^{15} \mathrm{~N}$ ratios found in V2676 Oph are similar to those of pre-solar grains with low ${ }^{12} \mathrm{C} /{ }^{13} \mathrm{C}$ and low ${ }^{14} \mathrm{~N} /{ }^{15} \mathrm{~N}$ ratios. Thus, the spectroscopic observations of V2676 Oph have provided the first observational evidence in support of the hypothesis that our solar system consists in part of materials formed in classical novae. On the other hand, strong CO emission bands were successfully detected in V2676 Oph [51]. The observed emission spectrum of $\mathrm{CO}$ (Figure 10) seems good enough to enable the determination of the isotopic ratio of carbon, for example, as in the case of V2274 Cyg [48]. Future comparisons of carbon isotopic ratios determined from $\mathrm{C}_{2}$ and $\mathrm{CO}$ are critically important for checking the accuracies of those values.

We can also compare the observed isotopic ratios of carbon and nitrogen in V2676 Oph with theoretical predictions $[29,10]$. Based on [29], the observed ${ }^{14} \mathrm{~N} /{ }^{15} \mathrm{~N}$ ratio in V2676 Oph can be reproduced by WDs having relatively heavy masses such as $1.0-1.25 \mathrm{M}_{\odot}$ (for either CO WD or $\mathrm{ONe}$ WD), while the predicted ${ }^{12} \mathrm{C} /{ }^{13} \mathrm{C}$ ratios seem too small in comparison with the observed ${ }^{12} \mathrm{C} /{ }^{13} \mathrm{C}$ ratio in V2676 Oph (and also compared to the values found in pre-solar grains). According to [10], a CO WD with $1.15 \mathrm{M}_{\odot}$ could be responsible for the observed isotopic ratios in V2676 Oph. Basically, higher masses for the WD component of V2676 Oph are necessary in order to explain the observed ${ }^{14} \mathrm{~N} /{ }^{15} \mathrm{~N}$ ratio, because more abundant ${ }^{15} \mathrm{~N}$ is produced in TNRs with higher temperatures and longer durations [29]. As discussed in Section 2, however, the slow evolution of the optical lightcurves of V2676 Oph indicates a low-mass WD component in this nova, $\sim 0.6 \mathrm{M}_{\odot}$, probably a CO WD. The absence of [Ne II] emission in the mid-infrared spectra and the relatively small ejected mass of V2676 Oph support this hypothesis. This discrepancy in the WD mass for V2676 Oph presents an interesting challenge for nucleosynthesis based on TNR theory. Thus, the mass of the WD component of V2676 Oph is still unknown (Table 4) and remains to be investigated from the viewpoint of nucleosynthesis in classical novae. The WD component of V2676 Oph is considered as CO WD based on the observations, and its mass is unclear unless photometric and spectroscopic monitoring observations of the binary system during the quiet phase of V2676 Oph can be performed (e.g., DQ Her [26]). 


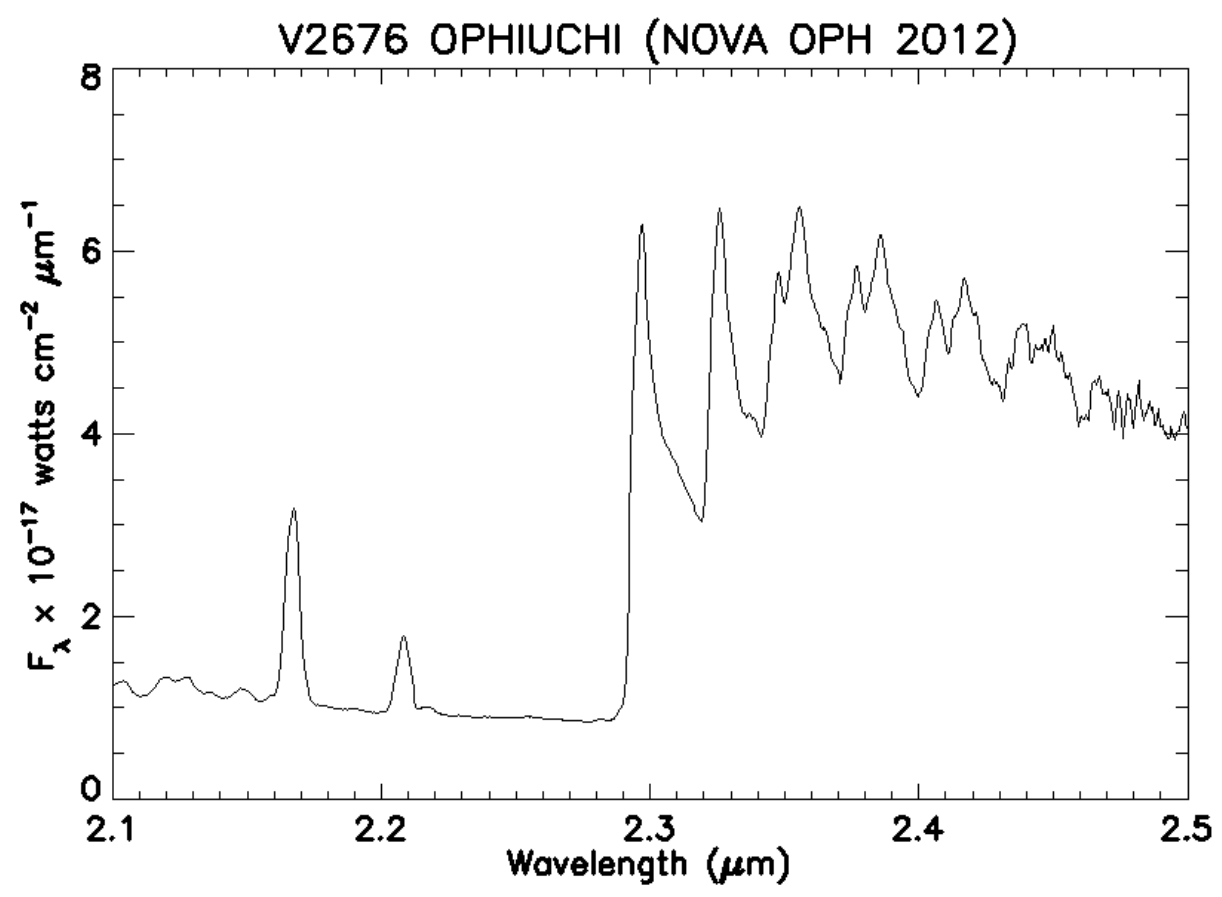

Figure 10: Emission spectrum of the first-overtone band of CO in V2676 Oph on UT 2012 May 1 (Rudy 2017, private communication).

\section{Summary}

The dust-forming nova V2676 Oph is a unique classical nova, because several kinds of diatomic molecules $\left(\mathrm{C}_{2}, \mathrm{CN}, \mathrm{CO}\right)$ were detected during the near-maximum and early-decline phases of the nova, and HAC grains (or PAH molecules) were also detected after dust formation. In particular, for the first time for novae, both the ${ }^{14} \mathrm{~N} /{ }^{15} \mathrm{~N}$ and ${ }^{12} \mathrm{C} /{ }^{13} \mathrm{C}$ ratios were determined simultaneously from the molecular-absorption-band profiles of $\mathrm{C}_{2}$ and $\mathrm{CN}$. Those ratios are similar to the ratios found in some type of pre-solar grains. The WD from which the nova originated is considered as CO WD, although its mass is still unknown $\left(\sim 0.6 \mathrm{M}_{\odot}\right.$ from the optical lightcurves and spectra, in contrast to $\sim 1.2 \mathrm{M}_{\odot}$ from the observed isotopic ratios of carbon and nitrogen). The geometry of the nova ejecta has been inferred from the emission-line profiles observed during the nebular phase of V2676 Oph; it is consistent with a slowly expanding $\left(\sim 750 \mathrm{~km} \mathrm{~s}^{-1}\right)$, equatorially concentrated ring of ejecta and a bipolar outflow.

\section{Acknowledgments}

The authors would like to thank Dr. Ashish Raj (Indian Institute of Astrophysics) for providing us the optical spectra of V2676 Oph published in his recent paper, and also thank Dr. Richard Rudy for providing us the CO emission spectrum of V2676 Oph prior to the publication of his data. We are also grateful to many amateur astronomers observing novae for discovery and photometric/spectroscopic monitoring of novae. Finally we would like to express our gratitude to 


\begin{tabular}{|c|c|c|c|c|}
\hline Observational feature & CO WD & ONe WD & $\begin{array}{c}\text { WD Mass } \\
{\left[\mathrm{M}_{\odot}\right]}\end{array}$ & References \\
\hline $\begin{array}{l}\text { (1) Slow decline during } \\
\text { the early-decline phase: } \\
0.013 \pm 0.04 \text { mag day }^{-1} \\
\text { in the } V \text {-band, indicating } \\
\text { a slow nova }\end{array}$ & Ok & $\mathrm{X}$ & $\sim 0.6$ & {$[23,26,22]$} \\
\hline $\begin{array}{l}\text { (2) Decrease in expansion } \\
\text { velocities from the pre- } \\
\text { maximum phase to the } \\
\text { maximum phase, followed } \\
\text { by increasing velocities } \\
\text { during the early-decline } \\
\text { phase }\end{array}$ & Ok & $\mathrm{X}$ & $0.5-0.7$ & {$[23,31,22]$} \\
\hline $\begin{array}{l}\text { (3) Total ejected mass: } \\
\sim 1.4 \times 10^{-5} \mathrm{M}_{\odot}\end{array}$ & Ok & $\mathrm{X}$ & $?$ & {$[47,20]$} \\
\hline $\begin{array}{l}\text { (4) No significant [Ne II] } \\
\text { emission at } 12.8 \mu \mathrm{m} \text { in } \\
\text { the nebular phase }\end{array}$ & Ok & $\mathrm{X}$ & $?$ & [34] \\
\hline $\begin{array}{l}\text { (5) Carbon-rich ejecta of } \\
\text { the nova: } \mathrm{C} / \mathrm{O}>1\end{array}$ & Ok & Ok & $?$ & {$[36,43,30]$} \\
\hline $\begin{array}{l}\text { (6) Low isotopic ratios of } \\
\text { both carbon and nitrogen: } \\
{ }^{12} \mathrm{C} /{ }^{13} \mathrm{C} \sim 4,{ }^{14} \mathrm{~N} /{ }^{15} \mathrm{~N} \sim 2\end{array}$ & Ok & Ok & $1.0-1.25$ & {$[32,29,10]$} \\
\hline
\end{tabular}

Table 4: Mass and other characteristics of the WD component of V2676 Oph.

Mr. Masayoshi Nagashima and Mrs. Tomoyo Kajikawa (Arai) for their efforts to find $\mathrm{C}_{2}$ and $\mathrm{CN}$ absorption bands in V2676 Oph during their student era.

\section{References}

[1] W.S. Adams, W.H. Christie, A.H. Joy, R.F. Sanford, \& O.C. Wilson, Radial Velocities from Absorption Lines in the Spectrum of Nova Herculis, PASP 47 (1935) 205

[2] L.I. Antipova, An Analysis of the CN Absorption Bands in the Spectrum of Nova Herculis 1934, SvA 13 (1969) 288

[3] A. Arai, \& M. Isogai, Nova Ophiuchi $2012=$ PNV J17260708-2551454, CBET 3072 (2012) 2

[4] A. Arai, H. Kawakita, Y. Shinnaka, \& A. Tajitsu, Origins of Absorption Systems of Classical Nova V2659 Cyg (Nova Cyg 2014), ApJ, 830 (2016) 30

[5] L.G. Althaus, A.M. Serenelli, A.H. Córsico, \& O.G. Benvenuto, Evolution of a 3-M $\odot$ star from the main sequence to the ZZ Ceti stage: the role played by element diffusion, MNRAS 330 (2002) 685 
[6] D. Barbier, \& D. Chalonge, Sur le spectre continu de Nova Herculis 1934 dans la région de courtes longueurs d'onde., Annales d'Astrophysique 3 (1940) 26 May 01, 2012

[7] D.P.K. Banerjee, \& N.M. Ashok, Near-infrared properties of classical novae: A perspective gained from Mount Abu Infrared Observatory, Bulletin of the Astron. Soc. of India 40 (2012) 243

[8] D.P.K. Banerjee, M.K. Srivastava, N.M. Ashok, \& V. Venkataraman, Near-infrared studies of the carbon monoxide and dust-forming Nova V5668 Sgr, MNRAS 455 (2016) L109

[9] R.K. Das, D.P.K. Banerjee, \& N.M. Ashok, Detection and evolution of the $C O(\Delta v=2)$ emission in Nova V2615 Ophiuchi (2007), MNRAS 398 (2009) 375

[10] P.A. Denissenkov, J.W. Truran, M. Pignatari, et al., MESA and NuGrid simulations of classical novae: CO and ONe nova nucleosynthesis, MNRAS 442 (2014) 2058

[11] A.M. Derdzinski, B.D. Metzger, \& D. Lazzati, Radiative shocks create environments for dust formation in classical novae, MNRAS 469 (2017) 1314

[12] R.A. Downes, \& H.W. Duerbeck, Optical Imaging of Nova Shells and the Maximum Magnitude-Rate of Decline Relationship, AJ 120 (2000) 2007

[13] A. Evans, \& J.M.C. Rawlings, PAH Emission in Novae / Polycyclic Aromatic Hydrocarbons, MNRAS 269 (1994) 427

[14] A. Evans, T.R. Geballe, J.M.C. Rawlings, \& A.D. Scott, Infrared spectroscopy of Nova Cassiopeiae 1993 - I. The pre-dust phase, MNRAS 282 (1996) 1049

[15] A. Evans, T.R. Geballe, J.M.C. Rawlings, S.P.S. Eyres, \& J.K. Davies, Infrared spectroscopy of Nova Cassiopeiae 1993 - II. Evolution of the dust, MNRAS 292 (1997) 192

[16] A. Evans, V.H. Tyne, O. Smith, T.R. Geballe, J.M.C. Rawlings, \& S.P.S. Eyres, Infrared spectroscopy of Nova Cassiopeiae 1993 - IV. A closer look at the dust, MNRAS 360 (2005) 1483

[17] A. Evans, \& J.M.C. Rawlings, Dust and molecules in nova environments, in Classical Novae, ed. M.F. Bode \& A. Evans (2nd ed., Cambridge Astrophysics Series No. 43; Cambridge: Cambridge Univ. Press) (2008) 308

[18] G.J. Ferland, D.L. Lambert, H. Netzer, D.N.B. Hall, \& S.T. Ridgway, Carbon monoxide emission and the Eta Carinae stage of Nova NQ Vulpeculae, ApJ 227 (1979) 489

[19] R.D. Gehrz, J.W. Truran, R.E. Williams, \& S. Starrfield, Nucleosynthesis in Classical Novae and Its Contribution to the Interstellar Medium, PASP 110 (1998) 3

[20] R.D. Gehrz, Infrared studies of classical novae, in Classical Novae, ed. M.F. Bode \& A. Evans (2nd ed., Cambridge Astrophysics Series No. 43; Cambridge: Cambridge Univ. Press) (2008) 167

[21] C.D. Gill, \& T.J. O’Brien, Emission-line profiles from model nova shells, MNRAS 307 (1999) 677

[22] P. Gil-Pons, E. García-Berro, J. José, M. Hernanz, \& J.W. Truran, The frequency of occurrence of novae hosting an ONe white dwarf, A\&A 407 (2003) 1021

[23] I. Hachisu, \& M. Kato, The UBV Color Evolution of Classical Novae. I. Nova-giant Sequence in the Color-Color Diagram, ApJ 785 (2014) 97

[24] I. Hachisu, \& M. Kato, A Light Curve Analysis of Classical Novae: Free-free Emission versus Photospheric Emission, ApJ 798 (2015) 76

[25] P.H. Hauschildt, S. Starrfield, \& F. Allard, Novae at Maximum Light: They can be Cool!, Cool Stars, Stellar Systems, and the Sun (ASP Conference Series 64), ed. J.-P. Caillault (San Francisco, CA: ASP) (1994) 705 
[26] K. Horne, W.F. Welsh, \& R.A. Wade, On the mass of nova DQ Hercules (1934), ApJ 410 (1993) 357

[27] T. Iijima, \& H. Nakanishi, Spectroscopic observations of the first helium nova V445 Puppis, A\&A 482 (2008) 865

[28] J. José, \& M. Hernanz, M., Nucleosynthesis in Classical Novae: CO versus ONe White Dwarfs, ApJ 494 (1998) 680

[29] J. José, \& M. Hernanz, The origin of presolar nova grains, M\&PS 42 (2007) 1135

[30] J. José, G.M. Halabi, \& M.F. El Eid, Synthesis of C-rich dust in CO nova outbursts, A\&A 593 (2016) 54

[31] M. Kato, \& I. Hachisu, Multiplicity of Nova Envelope Solutions and Occurrence of Optically Thick Winds, ApJ 699 (2009) 1293

[32] H. Kawakita, M. Fujii, M. Nagashima, M., et al., Formation of $C_{2}$ and $C N$ in nova V2676 Oph around its visual brightness maximum, PASJ 67 (2015) 17

[33] H. Kawakita, A. Arai, \& M. Fujii, The evolution of photospheric temperature in nova V2676 Oph toward the formation of $C_{2}$ and $C N$ during its near-maximum phase, PASJ 68 (2016) 87

[34] H. Kawakita, T. Ootsubo, A. Arai, Y. Shinnaka, \& M. Nagashima, Mid-infrared Spectroscopic Observations of the Dust-forming Classical Nova V2676 Oph, AJ 153 (2017) 74

[35] P.G. Martin, Overview of Nova DQ Her 1934, in Classical Novae, ed. M.F. Bode \& A. Evans (New York: Wiley) (1989) 93

[36] M. Nagashima, A. Arai, T. Kajikawa, H. Kawakita, E. Kitao, T. Arasaki, G. Taguchi, \& Y. Ikeda, The Transient Molecular Envelope in the Outflow of the Nova V2676 Oph during its Early Phase, ApJ 780 (2014) L26

[37] M. Nagashima, A. Arai, T. Kajikawa, H. Kawakita, E. Kitao, T. Arasaki, G. Taguchi, \& Y. Ikeda, Detection of Diatomic Molecules in the Dust Forming Nova V2676 Oph, Acta Polytechnica CTU Proc. 2 (2015) 212

[38] T. Nelson, K. Mukai, J. Sokoloski, L. Chomiuk, M. Rupen, \& A. Mioduszewski, Swift X-ray and UV observations of Nova Oph 2012, ATel 4055 (2012a) 1

[39] T. Nelson, K. Mukai, J. Sokoloski, L. Chomiuk, M. Rupen, \& A. Mioduszewski, Radio, X-ray and UV observations of Nova Oph 2012, ATel 4087 (2012b) 1

[40] H. Nishimura, Nova Ophiuchi $2012=$ Pnv J17260708-2551454, CBET 3072 (2012) 1

[41] L.R. Nittler, Presolar Stardust In The Solar System: Recent Advances for Nuclear Astrophysics, in Proceedings of the 10th Symposium on Nuclei in the Cosmos (NIC X), 13 (2008) http://pos.sissa.it/cgi-bin/reader/conf.cgi?confid=53

[42] A. Özdönmez, E. Ege, T. Güver \& T. Ak, A new catalogue of Galactic novae: investigation of the MMRD relation and spatial distribution, MNRAS 476 (2018) 4162

[43] M. Pontefract, \& J.M.C. Rawlings, The early chemical evolution of nova outflows, MNRAS 347 (2004) 1294

[44] A. Raj, N.M. Ashok, D.P.K. Banerjee, U. Munari, P. Valisa, \& S. Dallaporta, V496 Scuti: an Fe II nova with dust shell accompanied by CO emission, MNRAS 425 (2012) 2576

[45] A. Raj, D.P.K. Banerjee, N.M. Ashok \& S.C. Kim, Near-infrared studies of nova V5584 Sgr in the pre-maximum and early decline phase, RAA 15 (2015) 993 
[46] A. Raj, R.K. Das, \& F.M. Walter, Optical and Near-infrared Study of Nova V2676 Oph 2012, ApJ 835 (2017) 274

[47] A. Raj, M. Pavana, U.S. Kamath, G.C. Anupama, \& F.M. Walter, V2676 Oph: Estimating Physical Parameters of a Moderately Fast Nova, ACTA ASTRONOMICA 68 (2018) 79

[48] R.J. Rudy, W.L. Dimpfl, D.K. Lynch, et al., Near-Infrared Spectrophotometry and the CO Emission in V2274 Cygni (Nova Cygni 2001 No. 1), ApJ 596 (2003) 1229

[49] R.J. Rudy, T.R. Prater, R.C. Puetter, R.B. Perry, \& K. Baker, V496 Scuti, IAUC 9099 (2009) 1

[50] R.J. Rudy, E.A. Laag, K.B. Crawford, R.W. Russell, R.C. Puetter, \& R.B. Perry, Nova Ophiuchi 2012, CBET 3081 (2012a) 1

[51] R.J. Rudy, R.W. Russell, M.L. Sitko, C.M. Lisse, J. Rayner, W. Golisch, \& C. Chen, Nova Ophiuchi 2012, CBET 3103 (2012b) 1

[52] R.J. Rudy, R.W. Russell, W.L. Dimpfl, T.R. Prater, L. Bernstein, R.C. Puetter, R.B. Perry, M.L. Sitko, K.S. Bjorkman, \& J.P. Wisniewski, Carbon Monoxide Emission in the Nova V496 Scuti, Bulletin of American Astronomical Society, AAS Meeting 219 (2012c) 436.18

[53] R.J. Rudy, E.A. Laag, K.B. Crawford, M.C. Owens, M.S.L. Moody, R.C. Puetter, R.B. Perry, S. Baroni, \& E. Broens, V339 Delphini, CBET 3650 (2013) 1

[54] R.F. Sanford, The Cyanogen Bands in the Visual Region of the Spectrum of nova Herculis, PASP 47 (1935) 209

[55] M.M. Shara, T. Doyle, T.R. Lauer, et al., A Hubble Space Telescope Survey for Novae in M87. II. Snuffing out the Maximum Magnitude-Rate of Decline Relation for Novae as a Non-standard Candle, and a Prediction of the Existence of Ultrafast Novae, ApJ 839 (2017) 109

[56] C. Sneden, \& D. Lambert, Isotopic abundance ratios for carbon and nitrogen in Nova Herculis 1934, MNRAS 170 (1975) 533

[57] S. Starrfield, Thermonuclear processes, in Classical Novae, ed. M.F. Bode \& A. Evans (2nd ed., Cambridge Astrophysics Series No. 43; Cambridge: Cambridge Univ. Press) (2008) 77

[58] R.H. Stoy, \& A.B. Wyse, The Spectrum of Nova Herculis, 1934, PASP 47 (1935) 50

[59] R.J. Strope, B.E. Schaefer, \& A.A. Henden, Catalog of 93 Nova Light Curves: Classification and Properties, ApJ 140 (2010) 34

[60] J. Tanaka, D. Nogami, M. Fujii, K. Ayani, \& T. Kato, On the Rebrightenings of Classical Novae during the Early Phase, PASJ 63 (2011a) 159

[61] J. Tanaka, D. Nogami, M. Fujii, K. Ayani, T. Kato, H. Maehara, S. Kiyota, \& K. Nakajima, Spectral Evolution of the Unusual Slow Nova V5558 Sagittarii, PASJ 63 (2011b) 911

[62] W.P. Varricatt, T. Carroll, J. Ehle, \& T. Wold, Near-IR photometric monitoring observations of Nova Oph 2012 ATel $\mathbf{5 0 9 0}$ (2013) 1

[63] F.M. Walter, A. Battisti, S.E. Towers, H.E. Bond, \& G.S. Stringfellow, The Stony Brook/SMARTS Atlas of (mostly) Southern Novae, PASP 124 (2012) 1057

[64] B. Warner, Properties of novae; an overview, in Classical Novae, ed. M.F. Bode \& A. Evans (2nd ed., Cambridge Astrophysics Series No. 43; Cambridge: Cambridge Univ. Press) (2008) 16

[65] R. Wichmann, J. Krautter, K. Kawara, \& R.E. Williams, Near-infrared spectroscopy of nova V842 Centauri 1986, AGAb 5 (1990) 17 
[66] R. Wichmann, J. Krautter, K. Kawara, \& R.E. Williams, Near IR Spectroscopy of Nova V842 Cen 86, The Infrared Spectral Region of Stars, Proceedings of the International Colloquium held in Montpellier (France, 16-19 October, 1990), Edited by C. Jaschek \& Y. Andrillat. (Cambridge, UK: Cambridge University Press) (1991) 353

[67] R.E. Williams, The formation of novae spectra, AJ 104 (1992) 725

[68] R. Williams, \& E. Mason, Novae ejecta as colliding shells, Ap\&SS 327 (2010) 207

[69] O.C. Wilson, \& P.W. Merrill, Cyanogen Absorption in the Spectrum of Nova Herculis, 1934, PASP 47 (1935) 53

[70] A.B. Wyse, The Spectrum of Nova Aquilae (1918) Right Ascension: $18^{\mathrm{h}} 43^{\mathrm{m}} .8$; Declination: $+0^{\circ} 28^{\prime}$ (1900), Publications of Lick Observatory 14 (1940) 93 